\title{
THE METAL-RICH DISK POPULATION
}

Oxygen abundances

\author{
SOFIA FELTZING AND BENGT GUSTAFSSON \\ Astronomiska observatoriet \\ Uppsala, Sweden
}

Edvardson et al 1993 demonstrated that rapid build up of oxygen in the inner Galaxy. Do these high $[\mathrm{O} / \mathrm{Fe}]$ ratios prevail also for metal rich stars? We have obtained high resolution $(R=100000)$ spectra with the 2D-Coude Spectrometer at the McDonald Observatory $2.7 \mathrm{~m}$ telescope for 50 metal rich $\mathrm{F}, \mathrm{G}$ and $\mathrm{K}$ dwarfs. These stars where chosen to represent both an old stellar population which originates 'inside' the solar orbit and the young extreme Population I.

The oxygen abundances for all stars, except the stars in common with Barbuy and Grenon (1980), follow the same trends as in Edvardsson et al.(1993). Barbuy and Grenon (1980) studied 11 metal-rich dwarf stars on highly excentric orbits. For these we found lower iron abundances (typically 0.2 dex) and somewhat higher oxygen abundances. This means that the stars still stand out from the "normal" trend (see Fig. 1 in Barbuy and Grenon 1980) between oxygen and iron. What is special with the Barbuy and Grenon stars? The results for our samples give no clues to the origin of these stars or to the widely different oxygen abundances derived from different oxygen criteria. Their abundances might be due to erroneous $\mathrm{T}_{\text {eff }}$ or $\log (\mathrm{g})$. A change in $\mathrm{T}_{\text {eff }}$ by $-400 \mathrm{~K}$ or by -0.3 in $\log (\mathrm{g})$, is however required in this case.

The absence of a gradient in $[0 / F e]$ vs the $V_{L S R}$ velocity suggests the existence of an efficient radial mixing in the gas disk as compared to the star formation rate. The full study, including abundances for $\alpha^{-}, \mathrm{s}^{-}$and $\mathrm{r}$-process elements, will be presented in Feltzing and Gustafsson (1995).

\section{References}

Edvardsson, B., Andersen, J., Gustafsson, B., Lambert, D.L., Nissen, P.E. and Tomkin, J. (1993) $A \& A, \mathbf{2 7 5}, 101$

Feltzing, S. and Gustafsson, B. (1995), in preparation

Barbuy, B. and Grenon, M. (1990) in ESO/CTIO Workshop on "Bulges of Galaxies", ed. Jarvis, B.J. and Terndrup, D.M., p 83 